\title{
Ueber die quantitative Bestimmung des Glycogens.
}

\author{
Vorläufige Mittheilung. \\ Von
}

E. Salkowski.

(Aus dem chemischen Laboratorium des pathologischen Instituts zu Berlin.)

(Der Redaction zugegangen am 4. August 1902.)

Den Gehalt einer Leber an Glycogen festzustellen, galt früher als eine verhältnissmässig leichte und einfache Aufgabe. Seitdem aber Pflüger eine Fehlerquelle bei der Külz'schen Methode aufgefunden und verbesserte Methoden theils allein, theils in Verbindung mit Nerking angegeben hat, gehört die quantitative Bestimmung des Glycogens zu den allerunangenehmsten und langwierigsten analytischen Operationen. Nun hat Nerking selbst aber noch gefunden, dass bei dem, auch den neueren Methoden zu Grunde liegenden, langdauernden Zerkochen der Organe mit Kalilauge, das, nebenbei bemerkt, doch wohl nie ganz vollständig gelingt, die Quantität des Glycogens in ganz regelloser Weise bald zunimmt, bald abnimmt, ohne dass man im Stande ist, die Bedingungen für das eine oder andere Verhalten anzugeben. ${ }^{1}$ ) Damit ist auch die theoretische Grundlage der Külz-Pflüger'schen und Külz-Pflüger-Nerking'schen Methode erschüttert.

Diese Schwierigkeiten fallen nun theils ganz fort, theils sind sie ausserordentlich vermindert, wenn man sich davon freimacht, die Leber in frischem Zustand zu extrahiren oder zu zerkochen, sie vielmehr einer vorbereitenden Behandlung

1) Nach den neuesten Angaben von Pflüger soll dieses nun wieder nicht der Fall sein, das Glycogen vielmehr nicht angegriffen werden. Pflüger's Arch., Bd. 90, S. 523. 
unterwirft. Diese vorbereitende Behandlung besteht darin, dass man die Leber, in geeigneter Weise zerkleinert, mit absolutem Alkohol, dann mit Aether extrahirt und sie so in ein feines Pulver umwandelt. Ein Verlust an Glycogen ist dabei kaum zu befürchten, übrigens aber steht nichts im Wege, den Alkoholauszug zu verdunsten, die Quantität des darin enthaltenen Zuckers zu bestimmen und auf Glycogen umzurechnen. ${ }^{1}$ )

Das bei sorgfältigem Arbeiten erhaltene Leberpulver unterscheidet sich in sehr vortheilhafter Weise von der frischen Leber. Es löst sich verhältnissmässig leicht, jedenfalls sehr viel leichter als die frische Leber, in 2-3\% iger Kalilauge. Die Schnelligkeit der Auflösung hängt ganz davon ab, inwieweit die Pulverisirung der Leber gelungen ist. Ist sie besonders gut gelungen, so ist die Auflösung schon in wenigen Minuten beendigt. Die Lösung ist bräunlich gefärbt, aber viel klarer und dünnflüssiger als die aus der Leber direct erhaltene; ganz klar kann sie nicht sein, schon aus dem Grunde nicht, weil der phosphorsaure Kalk ungelöst bleibt.

Man braucht natürlich nur verhältnissmässig kleine Mengen, 5-10 g, des Leberpulvers zum Versuch zu nehmen.

Die alkalische Lösung, durch Absetzenlassen geklärt zweckmässig nimmt man nur einen aliquoten Theil, um das Filtriren zu vermeiden, also etwa $4 / 5$ - gibt, mit dem doppelten Volumen Alkohol gefällt, dann erst mit 60\% igem, dann starkem Alkohol etc. gewaschen, ein allerdings stark aschehaltiges Glycogen, das nur Spuren von Stickstoff enthält. Die Elementaranalyse ergab für das ca. 8 Stunden bei $105^{\circ}$ getrocknete Präparat, auf aschefreie Substanz berechnet, C $43,63^{\circ} \%$, H $6,61 \%{ }^{2}$ )

Glycogen von der Formel $\mathrm{C}_{6} \mathrm{H}_{10} \mathrm{O}_{5}$ erfordert $44,44 \% \mathrm{C}$ und $6,17 \% \mathrm{H}_{2} \mathrm{O}$, von der Formel $\left(\mathrm{C}_{6} \mathrm{H}_{10} \mathrm{O}_{5}\right)_{6}+\mathrm{H}_{2} \mathrm{O}$ (Huppert) $43,63 \%$ G und 6,26 .

1) Wohlgemuth hat von der vorbereitenden Behandlung der Leber bei der Glycogenbestimmung bereits Gebrauch gemacht. Diese Zeitschr., Bd. XXXV, S. 575.

2) 0,2066 $\mathrm{g}$ nach Abzug von $0,0130 \mathrm{~g}$ Asche im Schiffchen = $0,1936 \mathrm{~g}$ gab $0,3097 \mathrm{CO}_{2}$ und $0,0150 \mathrm{H}_{2} \mathrm{O}$. 
Trotz der guten Uebereinstimmung ist das Glycogen nicht ganz rein, denn es löste sich nicht ganz klar und die Lösung gab mit Brücke'schem Reagens eine, wenn auch nur geringe, Trübung, es steht ja aber nichts im Wege, das erhaltene Glycogen nach dem Vorgange Pflüger's zur Bestimmung zu hydrolysiren. Die Fällung mit Alkohol aus der alkalischen Lösung ist schon von Lebbin empfohlen worden, von Pflüger ist die Fällung unter Zusatz von Jodkalium eingeführt, der Nutzen dieses Zusatzes aber, soviel mir erinnerlich, nicht näher begründet worden. Zweifellos hat Pflüger die Angabe von Lebbin nicht gekannt.

Auch in künstlichem Magensaft löst sich die so vorbereitete Leber sehr schnell. Bei Anwendung von $5 \mathrm{~g}$ des Pulvers fand ich weder in dem bei der Verdauung nach 46 Stunden bleibenden Rückstand, noch in dem beim Aufkochen der nahezu neutralisirten Lösung entstandenen Coagulum Glycogen, auch nicht in Spuren. Aus dem auf etwa $150 \mathrm{ccm}$. eingedampften Filtrat fällte das doppelte Volumen Alkohol aschehaltiges Glycogen aus - weit weniger aschehaltig als das aus der alkalischen Lösung - , das nur Spuren von Stickstoff enthielt und dessen Lösung durch Brücke'sches Reagens + Salzsäure nicht gefällt wurde.

Die Elementaranalyse dieses Glycogens ergab $44,46 \% \mathrm{C}$ und $6,40 \% \mathrm{H},{ }^{1}$ ) Zahlen, die besser mit der Formel $\mathrm{C}_{6} \mathrm{H}_{10} \mathrm{O}_{5}$ übereinstimmen.

In vielen Fällen wird man aber auf die Isolirung des Glycogens ganz verzichten, dasselbe vielmehr sofort in Traubenzucker überführen und diesen bestimmen können. Dabei besteht nur eine Schwierigkeit: man muss die Abspaltung von Hexosederivaten, namentlich Chitosamin (Glucosamin), aus den Eiweisskörpern der Leber und von Pentosen aus den Nucleoproteiden vermeiden, auf das Jecorin braucht man keine Rücksicht zu nehmen, da es durch die vorangegangene Behandlung entfernt ist.

1) $0,1940 \mathrm{~g}$ nach Abzug von $0,0084 \mathrm{~g}$ Asche im Schiffchen $=$ $0,1853 \mathrm{~g}$ gab $0,3026 \mathrm{CO}_{2}$ und $0,1069 \mathrm{H}_{2} \mathrm{O}$. Die Analysen sind von Herrn Dr. C. Neuberg ausgeführt. 
Am nächsten liegt für diesen Zweck die Behandlung mit Fermenten. In der That gelingt es, sowohl durch Behandlung mit Speichel als auch mit käuflicher Diastase das Glycogen so vollständig zu extrahiren, dass der Rückstand kein Glycogen mehr enthält - auch bei sehr glycogenreicher Leber -, wenigstens ist mir das mit kleinen Mengen von $1 \mathrm{~g}$ gelungen. Damit entfallen auch, wie mir scheint, die von verschiedenen Seiten geäusserten Vermuthungen, dass das Glycogen in der Leber nicht als solches vorhanden sei, sondern gebunden, z. B. an Eiweisskörper. Die erhaltenen Lösungen müssen dann mit Säuren behandelt werden, zur Ueberführung der Zwischenstufen der Hydrolyse in Glucose.

Auch die Anwendung von verdünnter Milchsäure oder W einsäure unter Druck zur Ueberführung des Glycogens in Glycogendextrin und nachfolgende Behandlung mit Salzsäure nach Analogie der Amylumbestimmung oder Behandlung mit Salzsäure allein in abgestuften Verdünnungen kämen in Betracht. Welcher von diesen Wegen der empfehlenswertheste ist, bildet den Gegenstand weiterer Untersuchungen.

Selbstverständlich muss die Gewichtsbeziehung zwischen der Leber und dem Leberpulver bekannt sein und darin liegt unleugbar eine gewisse Schwierigkeit. Vermuthlich werden sich auch andere Organe auf diesem Wege auf Glycogengehalt untersuchen lassen.

Als ein weiterer einfacherer Weg zur Bestimmung des Glycogens würde sich vielleicht auch die Autodigestion (Autolyse) der Leber eignen, bei welcher, wie ich seiner Zeit gefunden habe, das Glycogen völlig verschwindet und zwar unter Bildung von Zucker bezw. reducirenden Substanzen, doch bedarf es noch einer Reihe von Versuchen, ehe hierüber ein bestimmtes Urtheil möglich ist. Dabei würde der präformirte Zucker in der Leber - wenn er überhaupt existirt - mitbestimmt werden. 